\title{
Der Einfluß einer Röntgen-Ganzkörperbestrahlung auf den Einbau von 2-14C-Glycin in die Proteine der Leber, Milz und des Blutes von Ratten
}

\author{
H. MaAss, E. JenNer und F. Hölzel * \\ Universitäts-Frauenklinik Hamburg-Eppendorf (Dir.: Prof. Dr. med. K. Tномsen) \\ (Z. Naturforschg. 22 b, 634-638 [1967] ; eingegangen am 20. Dezember 1966)
}

\begin{abstract}
Whole body irradiation with $800 \mathrm{R}$ caused a three fold increase of radioactivity in the liver and spleen proteins of rats. Analysis of the radioactivity of the precursor pool showed an increase of ${ }^{14} \mathrm{C}$-activity to the same degree as found for the proteins. Thus the increase of the incorporation rate of ${ }^{14} \mathrm{C}$-glycine into the proteins is simulated, and consequently there is no change of the protein synthesis in the liver of an irradiated rat as measured with ${ }^{14} \mathrm{C}$-glycine. This is probably true for the rat spleen also.
\end{abstract}

In früheren Untersuchungen konnte gezeigt werden, daß der Gehalt an Nucleosidphosphaten in Niere, Leber, Milz, Thymus und Blut von Ratten nach einer Röntgen-Ganzkörperbestrahlung mit $800 \mathrm{R}$ charakteristische Veränderungen aufweist (MaAss ${ }^{1}$ ). Im Prinzip kam es in allen untersuchten Organen zu einer Abnahme der Nucleosidtriphosphate in bestimmten Zeitabschnitten nach der Bestrahlung. Die Nucleosidmono- und -diphosphate verhielten sich demgegenüber gegensinnig.

Die stärksten Veränderungen fanden sich vier bis sechs Tage nach einer Ganzkörperbestrahlung. In weiteren Versuchen sollte die Frage geklärt werden, inwieweit die nachgewiesene Störung des Energiehaushaltes der untersuchten Organe eine Konsequenz für die Proteinsynthese hat. Dazu wurde der Einbau von $2 \cdot{ }^{14} \mathrm{C}$-Glycin in das Organprotein zu verschiededen Zeitpunkten nach einer Bestrahlung mit ebenfalls $800 \mathrm{R}$ untersucht. Untersuchungen zum Einfluß einer Ganzkörperbestrahlung auf den Einbau markierter Aminosäuren in Organoproteine liegen bereits mehrfach vor. In keinem Fall wurde jedoch parallel nach Veränderungen des Aminosäurepools bzw. seiner Markierung in den bestrahlten Tieren gefahndet. Neben der Messung der Incorporierungsrate des ${ }^{14} \mathrm{C}$-Glycin wurde daher die Menge an ungebundenem Glycin sowie der Anteil des ${ }^{14} \mathrm{C}$-Glycin am Glycinpool in der Leber der bestrahlten und unbestrahlten Tiere untersucht.

\section{Material und Methodik}

Für die Versuche wurden $80 \mathrm{~g}$ schwere männliche Wistar-Ratten eines ingezüchteten Stammes verwendet.

\footnotetext{
* Jetzt H.-Pette-Institut f. exp. Virologie und Immunologie an der Universität Hamburg.

1 H. MaAss, Z. ges. exp. Med. 133, 97 [1960].
}

Die Tiere erhielten während der Versuchsdauer Trokkenfutter und Wasser ad libitum.

Die Bestrahlung der Tiere erfolgte mit einer 200KV-Röntgenstrahlung bei $0,5 \mathrm{~mm}$ Kupferfilterung. Zur Messung der ${ }^{14} \mathrm{C}$-Glycin-Incorporierungs-Rate in die Organproteine wurden $5 \mu \mathrm{C} \cdot 2{ }^{14} \mathrm{C}$-Glycin $(3,9 \mu \mathrm{C} / \mu \mathrm{Mol})$ pro $100 \mathrm{~g}$ Ratte intraperitoneal injiziert und die Tiere nach 15, 30, 60, 120 und 180 Min. durch Nackenschlag und Ausblutenlassen aus den Karotiden getötet. Eine Blutmenge sowie die Organproben wurden möglichst rasch entnommen und in $5 \mathrm{ml}$ einer 6-proz. $\mathrm{HClO}_{4}$ mit Hilfe des Ultraturrax in einer Kältemischung homogenisiert. Vom Töten der Tiere bis zum Enteiweißen der Gewebshomogenate vergingen 3 bis 4 Minuten. Das Sediment wurde zunächst mehrfach mit $\mathrm{HClO}_{4}$ und 4-proz. Trichloressigsäure gewaschen und schließlich in Anlehnung an die Angaben von Siekevitz ${ }^{2}$ weiter aufgearbeitet. Die Proteinproben wurden schließlich in Aceton aufgenommen und auf Aluminiummeßschälchen gegeben. Die Messung der Impulsraten erfolgte in einem Methandurchflußzähler der Firma Friesecke \& Höpfner, Erlangen. Die Impulse der gemessenen Proben wurden unter Berücksichtigung der Eigenabsorption für ${ }^{14} \mathrm{C}$ auf $\mathrm{mg}$ Protein bezogen.

Die Bestimmung des freien Glycins in der Leber erfolgte nach Alexander et al. ${ }^{3}$. Hierbei wird der nach Decarboxylierung und Desaminierung mit Ninhydrin entstehende Formaldehyd destilliert und mit Chromotropsäure (1.8-Dihydroxynaphthalin-3.6-Disulfonsäure) umgesetzt. Die Messung der Farbintensität erfolgte bei $578 \mathrm{~nm}$ im Photometer Eppendorf. Die Menge an freiem Glycin wurde einer Eichkurve entnommen.

\section{Ergebnisse}

In der Abb. 1 sind die Incorporierungsraten des intraperitoneal injizierten ${ }^{14} \mathrm{C}$-Glycin in das Protein der Leber, Milz und des Blutes dargestellt. Wie aus

\footnotetext{
2 P. Siekevitz, J. biol. Chemistry 195, 549 [1952].

3 B. Alexander, G. Landwehr u. A. M. Seligmann, J. biol. Chemistry 160, 51 [1945].
} 


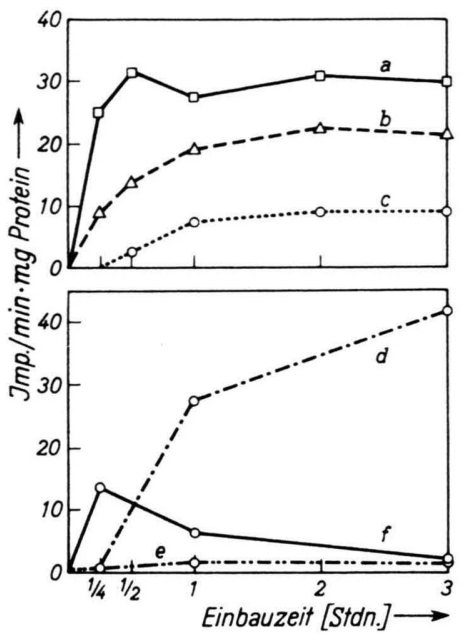

Abb. 1. Der Einbau von ${ }^{14} \mathrm{C}-$ Glycin in das Protein von Rattenleber, -Milz- und -Blut. $\square-\square$ Leber-Eiweiß, $\triangle \cdots \triangle$ Milz-

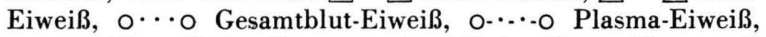
○ -.. ० Blutzell-Eiweiß, ०-૦ eiweißfreier Plasmaüberstand.

dem oberen Teil der Abbildung hervorgeht, kommt es zu einer raschen Aufnahme der ${ }^{14} \mathrm{C}$-Aktivität in das Protein der Leber. Das Maximum der Incorporierung war bereits eine halbe Stde. nach der intraperitonealen Injektion erreicht. Danach bildete sich für weitere drei Stdn. ein Plateau aus. In der Milz war die Zunahme der ${ }^{14} \mathrm{C}$-Aktivität zögernder, so daß maximale Werte erst zwei Stdn. nach der Injektion gemessen wurden. Über die Aktivitätsverhält. nisse im Blut gibt der untere Teil der Abbildung Auskunft. Wie die ausgezogene Linie erkennen läßt, sinkt die Radioaktivität im eiweißfreien Plasmaüberstand nach der ersten Messung 15 Min. nach der Injektion sehr rasch ab. Auffällig früh kommt es jedoch auch im Plasmaeiweiß zu einer Zunahme der Radioaktivität, während im Protein der zellulären Bestandteile praktisch keine ${ }^{14}$ C-Aktivität gemessen wurde. Die gering gemessenen Aktivitätswerte sind als Verunreinigung und als die noch mögliche Proteinsynthese in Retikulozyten und Leukozyten aufzufassen.

Bei den bestrahlten Tieren erfolgte die Entnahme der Organe jeweils 30 Min. nach Injektion des ${ }^{14} \mathrm{C}$ Glycin. Die Tiere wurden 14 und 24 Stdn. sowie 2, 4, 6 und 8 Tage nach einer Ganzkörperbestrahlung mit $800 \mathrm{R}$ getötet. Die Ergebnisse sind in der nächsten Abbildung (Abb. 2) zusammengefaßt. Neben der gemessenen Impulsrate ist für die Leberwerte die Standardabweichung mit eingezeichnet. In dem Beobachtungszeitraum von 8 Tagen kommt es überraschenderweise zu einer erheblichen Zunahme der

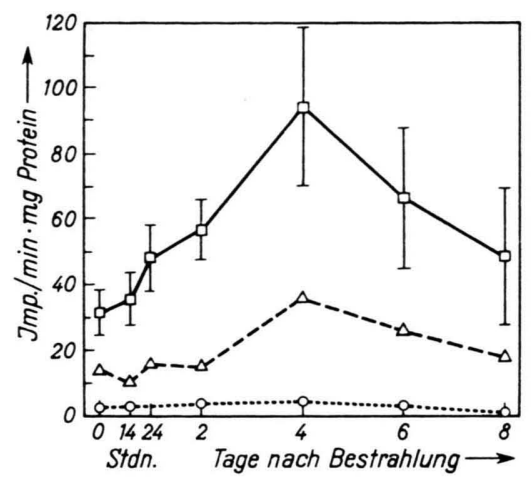

Abb. 2. Der Einfluß von Röntgenstrahlen (800 r) auf den Einbau von ${ }^{14} \mathrm{C}$-Glycin in das Protein von Rattenleber, -Milz und -Blut bei konstanter Einbauzeit von 30 Minuten. $\square-\square$ Leber-Eiweiß, $\triangle \cdots \triangle$ Milz-Eiweiß, $\circ \cdots \circ$ Gesamtblut-Eiweiß.

Incorporierungsrate des injizierten ${ }^{14} \mathrm{C}$-Glycin in die Proteine von Leber und Milz der bestrahlten Tiere. Das Maximum dieses Effektes findet sich vier Tage nach der Bestrahlung, d. h. genau zu dem Zeitpunkt, zu dem in früheren Untersuchungen das Minimum der Nucleosidtriphosphat-Konzentrationen gemessen wurde ${ }^{1}$. Die Zunahme der Radioaktivität im Lebereiweiß beträgt etwa das Dreifache, im Milzeiweiß das Zweieinhalbfache der bei den Kontrollen gemessenen ${ }^{14} \mathrm{C}$-Aktivitäten. Im Gesamtblut fanden sich erwartungsgemäß keine signifikanten Veränderungen.

Zur Klärung der Frage, ob es sich hierbei um echte Steigerungen der Incorporierungsraten handelt oder lediglich um Veränderungen des Glycinpools, wurde in der Leber die Menge des freien Glycins bestimmt. Wie die Tab. 1 erkennen läßt, fand sich pro g Frischleber bei den unbestrahlten Kontrollen $144 \mu \mathrm{g}$ Glycin, bei den Lebern der bestrahlten Tiere zum Zeitpunkt der maximalen ${ }^{14} \mathrm{C}$-Incorporierungsrate $174 \mu \mathrm{g}$. Es zeigt sich also kein eindeutiger Unterschied in der Poolgröße für freies Glycin in den Lebern bestrahlter und unbestrahlter Tiere. Der beobachtete Anstieg der Incorporierungsrate in das Protein der bestrahlten Tiere ist demnach nicht einfach durch Änderungen des Glycinpools erklärt.

\begin{tabular}{cc}
\hline unbestrahlte Kontrollen & $\begin{array}{c}4 \text { Tage nach Ganzkörper- } \\
\text { bestrahlung mit 800 R }\end{array}$ \\
\hline $144 \pm 24$ & $174 \pm 59$ \\
\hline
\end{tabular}

Tab. 1. Freies, mit schwefelsaurem Na-wolframat extrahiertes Glycin in Leberproben von männlichen Wistar-Ratten in $\mu \mathrm{g}$ Glycin/g Frischleber (Doppelwerte von je 4 Versuchstieren).

Um nähere Aussagen über den eigentlichen Umsatz des Glycins in der Leber zum Zeitpunkt des 
vermehrten Glycineinbaus 4 Tage nach Ganzkörperbestrahlung mit $800 \mathrm{R}$ machen zu können, wurde die Menge der freien, nicht proteingebundenen ${ }^{14} \mathrm{C}$-Aktivität in der Leber gemessen. Zunächst bestimmten wir bei drei Versuchstieren den Anteil des freien ${ }^{14} \mathrm{C}$-Glycin an der Gesamt- ${ }^{14} \mathrm{C}$-Aktivität im sauren Extrakt aus Lebergewebe 4 Tage nach der Bestrahlung, eine halbe Stde. nach intraperitonealer Injektion des $2{ }^{14} \mathrm{C}-G l y c i n$. Dazu wurde von jedem Tier eine Leberprobe mit 3-proz. $\mathrm{HClO}_{4}$ und eine andere mit dem bei dem Glycinpool beschriebenen Fällungsgemisch (schwefelsaures Natriumwolframat) extrahiert. Die ${ }^{14} \mathrm{C}$-Aktivität im neutralisierten perchlorsauren Extrakt aus dem Lebergewebe entspricht unter den genannten Bedingungen nur noch zu einem Teil dem freien ${ }^{14} \mathrm{C}$-Glycin in den Leberproben. Es ist anzunehmen, daß sich nach der verhältnismäßig langen Zeit von 30 Min. nach der Injektion eine Reihe ${ }^{14} \mathrm{C}$-markierter säurelöslicher Metabolite des Glycins neben dem noch vorhandenen nicht proteingebundenen ${ }^{14} \mathrm{C}$-Glycin in dem Extrakt befinden. Wenn man die mit schwefelsaurem $\mathrm{Na}$ triumwolframat gewonnenen Leberextrakte wie bei der Bestimmung des Glycinpools mit Ninhydrin behandelt und den dabei aus Glycin entstehenden Formaldehyd destilliert, findet man nur mehr ca. $10 \%$ der ${ }^{14} \mathrm{C}$-Aktivität des schwefelsauren Na-wolframat-Extraktes aus der Leber als ${ }^{14} \mathrm{C}$-Formaldehyd im Destillat wieder. Demnach muß man annehmen, daß nur $10 \%$ der Gesamt ${ }^{14} \mathrm{C}$-Aktivität im säurelöslichen Extrakt 30 Min. nach der Injektion noch als ${ }^{14} \mathrm{C}$-Glycin vorliegen, während der übrige Anteil bereits in Metabolite des Glycins umgewandelt wurde.

In der Tab. 2 sind die Werte für die ${ }^{14} \mathrm{C}$-Radioaktivität, die bei bestrahlten und unbestrahlten Tie-

\begin{tabular}{ccc}
$\mathrm{a}$ & $\mathrm{b}$ & $b / a$ \\
un- & 4 Tage & \\
bestrahlt & nach $800 \mathrm{R}$ & \\
\hline
\end{tabular}

\begin{tabular}{lccc}
\hline $\begin{array}{l}\text { C14-Aktivität in 100 mg } \\
\text { gereinigtem Leber- } \\
\text { protein (ca. 2000 mg }\end{array}$ & & & \\
$\begin{array}{l}\text { Frischleber) } \\
\text { C14-Aktivität im }\end{array}$ & 31500 & 93900 & 3,0 \\
$\begin{array}{l}\text { Protein von } 100 \mathrm{mg} \\
\begin{array}{l}\text { Frischleber } \\
\text { C14-Aktivität im }\end{array}\end{array}$ & 1575 & 4695 & 3,0 \\
neutralisierten & & & \\
$\begin{array}{l}\text { HClO } \\
100 \text { mg Extrakt aus }\end{array}$ & & & \\
\hline
\end{tabular}

Tab. 2. ${ }^{14} \mathrm{C}$-Radioaktivität $(\mathrm{IpM})$ in Rattenleber $30 \mathrm{Min}$. nach i.p.-Injektion von $0,4 \mathrm{ml} 2{ }^{14} \mathrm{C}$-Glycin $(10 \mu \mathrm{C} / \mathrm{ml}=$ $3,9 \mu \mathrm{C} / \mu \mathrm{Mol})$ pro Ratte $(80 \mathrm{~g})$ bei unbestrahlten Kontrollen sowie 4 Tage nach Ganzkörperbestrahlung mit $800 \mathrm{R}$. ren 30 Min. nach Injektion der radioaktiv-markierten Aminosäure im Leberprotein und im perchlorsauren Extrakt der Frischleber wiedergefunden wurden, zusammengestellt. Dabei wurde die ${ }^{14} \mathrm{C}$-Aktivität im perchlorsauren Extrakt aus Lebergewebe auf die bei den ${ }^{14} \mathrm{C}$-Glycin-Einbauversuchen (Abb. 2) verwendete $2 \cdot{ }^{14} \mathrm{C}$-Glycin-Injektionslösung $(10 \mu \mathrm{C} / \mathrm{ml}$ $=3,9 \mu \mathrm{Mol}$ ) umgerechnet.

Die ${ }^{14} \mathrm{C}$-Aktivitäten im Protein von $100 \mathrm{mg}$ Frischleber (mittlere Zeile der Tab. 2) betragen $5 \%$ der in 100 mg gereinigtem Leberprotein gemessenen Radioaktivität (obere Zeile der Tabelle), da $100 \mathrm{mg}$ Frischleber etwa $5 \mathrm{mg}$ gereinigtes Eiweiß enthalten. Die ${ }^{14} \mathrm{C}$-Radioaktivität im neutralisierten $\mathrm{HClO}_{4}$ Extrakt aus $100 \mathrm{mg}$ Frischleber beträgt $30 \mathrm{Min}$. nach Verabreichung des ${ }^{14} \mathrm{C}$-Glycins bei bestrahlten und unbestrahlten Tieren etwa 50\% der im Leberprotein gemessenen Radioaktivität. Die Tab. 2 zeigt, daß sich 4 Tage nach Ganzkörperbestrahlung der Tiere mit $800 \mathrm{R}$ eine etwa gleichgroße Erhöhung im Indikatorpool der Leber $\left({ }^{14} \mathrm{C}\right.$-Aktivität im perchlorsauren Extrakt) wie in der ${ }^{14} \mathrm{C}$-Markierung des Leberproteins findet, bei nahezu konstantem Glycinpool (s. Tab. 1).

Zur Kontrolle dieser Befunde wurde in einer weiteren Versuchsreihe bei den Kontrolltieren sowie den bestrahlten Tieren die ${ }^{14} \mathrm{C}$-Aktivität im neutralisierten perchlorsauren Leberextrakt während der ersten halben Stde. nach intraperitonealer Injektion von $4 \mu \mathrm{C} \quad 2-{ }^{14} \mathrm{C}$-Glycin untersucht. Hiermit ergibt sich ein Hinweis auf die Resorptionsverhältnisse des ${ }^{14} \mathrm{C}$-Glycins aus dem Peritonealraum; besonders sollte jedoch geprüft werden, ob sich Veränderungen in den bestrahlten Tieren erkennen lassen.

Die Ergebnisse sind in Abb. 3 zusammengefaßt. Die gestrichelte Kurve gibt die ${ }^{14} \mathrm{C}$-Aktivität im säurelöslichen Extrakt der Leber der Kontrolltiere wieder. Man erkennt, daß nach der Injektion ein rascher Anstieg der Radioaktivität erfolgt, der bereits nach 5 Min. sein Maximum erreicht. In der Leber der bestrahlten Tiere fand sich eine erheblich stärkere Aktivität mit einem Maximum von $20 \cdot 10^{3}$ IpM/100 mg Frischleber 10 Min. nach der Injektion. Die erhöhten Werte bleiben während des Versuchszeitraums von $30 \mathrm{Min}$. bestehen. Die Markierung des Glycinpools in der Leber der bestrahlten Tiere während der Incorporierungszeit von $30 \mathrm{Min}$., die für die Proteinsynthese-Versuche zugrundegelegt wurde, ist also deutlich gegenüber den Kontrollwerten erhöht. Es muß also geschlossen werden, daß 
die erhöhte Incorporierungsrate des ${ }^{14} \mathrm{C}$-Glycins in das Leberprotein der bestrahlten Tiere keine erhöhte Syntheseleistung repräsentiert, sondern lediglich auf die entsprechend höhere ${ }^{14} \mathrm{C}$-Markierung des Pools des säurelöslichen Glycins und seiner Metabolite zurückzuführen ist. Diese Aussage kann jedoch streng nur für die Leber gemacht werden, weil die Pooluntersuchungen an der Milz nicht durchgeführt wurden.

\section{Diskussion}

Ausgangspunkt dieser Untersuchungen war die Prüfung der Frage, inwieweit der früher von uns nachgewiesene Abfall des ATP-Gehaltes in Leber, Milz und Blut nach einer Ganzkörperbestrahlung mit $800 \mathrm{R}$ (MaAss ${ }^{1}$ ) eine Konsequenz für Aminosäureincorporierende Prozesse hat. Versuche an YoshidaAscites-Sarkomzellen hatten nämlich gezeigt, daß bei der in vitro-Incorporierung von ${ }^{14} \mathrm{C}$-Alanin in das Protein der Tumorzellen eine strenge Abhängigkeit vom ATP-Gehalt der Zellen bestand (Schubert et al. ${ }^{4}$ ). Die röntgenbestrahlten Tumorzellen zeigten nur so lange eine Hemmung des Aminosäureeinbaus in das Protein, solange auch ein durch die Glykolysehemmung bedingter ATP-Abfall nachweisbar war. Eine Beeinflussung anderer an der Proteinsynthese beteiligter Reaktionen fand sich jedenfalls nicht.

Untersuchungen über strahleninduzierte Veränderungen der Incorporierung verschiedener Aminosäuren in das Protein nach Ganzkörperbestrahlung sind bereits mehrfach durchgeführt worden. Die Ergebnisse sind uneinheitlich. Meistens sind die Incorporierungszeiten viel zu lang gewählt worden. Wie aus der Abb. 1 hervorgeht, stellte sich in der Leber bereits in einem Zeitraum zwischen einer viertel bis einer halben Stde. nach der Injektion ein Plateau ein. Exakte Hinweise auf die Incorporierungsrate und ihre evtl. Veränderung nach Röntgenbestrahlung lassen sich daher nur in diesem kurzen Zeitabschnitt gewinnen. Das nachgewiesene Plateau der ${ }^{14} \mathrm{C}$-Aktivität im Protein ist vermutlich nicht als Ausdruck eines Fließgleichgewichtes von Incorporierungsrate und Abbaurate aufzufassen. Wie aus der Überschlagsrechnung nämlich hervorgeht,

4 G. Schubert, G. Bettendorf, H. A. Künkel, H. Maass u. G. H. Rathgen, Strahlentherapie 106, 483 [1958].

5 D. L. Coleman u. M. E. Ashworth, Amer. J. Physiol. 197, 839 [1959].

6 W. Maurer, Dynamik des Eiweißes, 10. Colloq. d. Gesellsch. f. Physiolog. Chem. am 9./12. April 1959 in Mosbach/Baden. fand sich 30 Min. nach der Injektion nur mehr eine geringe Markierung des Glycinpools, der nur etwa $10 \%$ des in das Eiweiß eingebauten ${ }^{14} \mathrm{C}$-Glycins und seiner Metabolite ausmacht. Der größere Teil ist also bereits zu diesem Zeitpunkt anderweitig verwertet oder eliminiert. Ähnliche Ergebnisse fanden Cleman und Ashworth ${ }^{5}$. Das nachgewiesene Plateau ist offenbar Ausdruck einer relativ langen biologischen Halbwertszeit der Proteine (MAURer ${ }^{6}$ ).

Eine Hemmung der ${ }^{14} \mathrm{C}$-Glycin-Incorporierung in das Globin nach Ganzkörperbestrahlung mit $800 \mathrm{R}$ fanden Richmond et al. ${ }^{7}$ bei in vitro-Untersuchungen an Milzzellen. Supplee und Entenman ${ }^{8}$ beobachteten 24 Stdn. nach einer Bestrahlung mit $2500 \mathrm{R}$ bei Ratten eine durchschnittliche Zunahme des Lebergewichtes um etwa 30 Prozent. Daraufhin bestimm ten Payne et al. ${ }^{9}$ ebenfalls $24 \mathrm{Stdn}$. nach $2500 \mathrm{R}$ den Einbau von ${ }^{14} \mathrm{C}$-markiertem Formiat in das Cytoplasmaeiweiß sowie die ${ }^{32} \mathrm{P}$-Aufnahme in die Cytoplasma-RNS der Leberzellen. Die Autoren registrierten einen Aktivitätszuwachs im ${ }^{14} \mathrm{C}$-Protein um $34 \%$ und einen gleichsinnigen Anstieg der ${ }^{32} \mathrm{P}$-Aktivität der RNS. Ebenfalls eine Zunahme der Incorporierungsrate von $3 \cdot{ }^{14} \mathrm{C}$-DL-Phenylalanin fanden Butler, Cohn und Crathorn ${ }^{10}$ innerhalb der ersten Stde. nach einer Ganzkörperbestrahlung mit $500 \mathrm{R}$ in Kern-, Mitochondrien-, Mikrosomen- und Endüberstandfraktionen der Leber. Dagegen konnte Gould ${ }^{11}$ bei Verwendung von $2 \cdot{ }^{14} \mathrm{C}$-Phenylalanin 24 und 48 Stdn. nach Ganzkörperbestrahlung keine eindeutige Änderung der Proteinsynthese in der Rattenleber feststellen. Ebenfalls fand sich keine Beeinflussung des Aminosäureeinbaus in die Proteine des Thymus nach $500 \mathrm{R}$ (Butler et al. ${ }^{10}$ ). Bereits Hevesy ${ }^{12}$ hatte einen Anstieg der Incorporierungsrate von markiertem Natriumacetat in das Leberprotein nach Ganzkörperbestrahlung festgestellt. Untersuchungen des Glycinpools sind von den Autoren nicht durchgeführt worden. Es ist daher die Frage, ob die gemessene Steigerung der Incorporierungsraten als echt anzusehen ist.

Am besten vergleichbar mit unseren Versuchen sind die Untersuchungen von Ricotri und Notario ${ }^{\mathbf{1 3}}$.

\footnotetext{
7 J. E. Richmond, K. J. Altman u. K. Salomon, J. biol. Chemistry 190, 817 [1951].

${ }^{8}$ H. Supple u. C. Entenman, Federat. Proc. 11, 156 [1952].

9 A. H. Payne, L. S. Kelly u. C. Entenman, Proc. Soc. exp. Biol. Med. 81, 698 [1952].

10 J. A. V. Butler, P. Cohn u. A. R. Crathorn, Progress in Radiobiol., Oliver u. Boyd, Edinburgh 1957.
} 
Die genannten Autoren konnten feststellen, daß 5 Tage nach Bestrahlung mit 500 und $1000 \mathrm{R}$ eine reduzierte Incorporierung von ${ }^{14} \mathrm{C}$-Glycin in das Lebereiweiß von Wistar-Ratten auftrat. Die Untersuchung war 1 Stde. nach intraperitonealer Injektion der Aminosäure durchgeführt worden. Der Unterschied der Versuchsanordnung war vor allem der, daß die italienischen Autoren an der Carboxylgruppe markiertes $1-{ }^{14} \mathrm{C}-\mathrm{Glycin}$ verwandten. Es müßte geprüft werden, inwieweit es in diesen Versuchen zu einer Änderung des ${ }^{14} \mathrm{C}$-Glycinpools möglicherweise durch eine erhöhte Decarboxylierung gekommen ist.

Glycinpool-Bestimmungen wurden lediglich von Schreier et al. ${ }^{14,15}$ durchgeführt. Danach konnte bei Ratten nach $700 \mathrm{R}$ ein „Trend“ in Richtung auf eine Vergrößerung des Ausgangs-Pools für Glycin festgestellt werden.

Wie wir eindeutig nachweisen konnten, ist die gesteigerte Aufnahme von markiertem Glycin in das Leberprotein durch eine Höhermarkierung des Vorstufenpools vorgetäuscht. Einen ähnlichen Mechanismus möchten wir daher für die in der Literatur beschriebenen Einbausteigerungen annehmen. Demnach ist eine Beeinflussung der Proteinsynthese in Leber und Milz nach einer Bestrahlung mit $800 \mathrm{R}$ nicht nachzuweisen. Der stark erniedrigte ATP-Gehalt in beiden Organen am 4. Tag nach der Bestrahlung hat also keine Konsequenz für die Incorporierungsrate von ${ }^{14} \mathrm{C}$-Glycin und seiner in dem Versuchszeitraum gebildeten Metabolite. Entsprechend fanden Goetze und Goetze ${ }^{16}$ keine Veränderung des ${ }^{14} \mathrm{C}$-Glycin-Einbaus in das Protein von Leberschnitten aus bestrahlten Tieren.

Die Frage nach der Ursache der Höhermarkierung des Vorstufenpools ist zur Zeit nicht zu beantworten. Wie aus der Abb. 3 hervorgeht, ist die erhöhte ${ }^{14} \mathrm{C}$-Aktivität im perchlorsauren Extrakt aus den Lebern der bestrahlten Tiere bereits in den ersten Min. nach der Injektion nachweisbar. Wir möchten daher nicht annehmen, daß die Höhermarkierung als Folge eines vermehrten Zustroms von ${ }^{14}$ C-Glycin bzw. seiner Metabolite aus Organen zu

11 R. G. Gould, Los Alamos Sci. Lab. of the Univ. of California, LA-2199, 21. 8. 1958.

12 G. Hevesy, Nature [London] 164, 269 [1949].

13 V. Ricotri u. A. Notario, Minerva nucleare [Torino] 7, 18 [1963].

14 K. Schreier, K. I. Altman u. L. H. Hempemann, Proc. Soc. exp. Biol. Med. 87, 61 [1954].

15 K. Schreier, K. Wirth u. W. Hack, Hoppe-Seyler's Z. physiol. Chem. 304, 186 [1956].

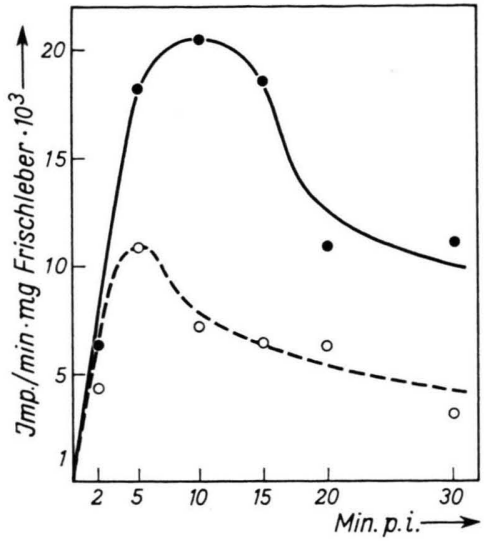

Abb. 3. ${ }^{14} \mathrm{C}$-Aktivität im neutralisierten perchlorsauren Extrakt aus $100 \mathrm{mg}$ Frischleber von $80 \mathrm{~g}$ schweren $\hat{\delta}$ Ratten, 4 Tage nach Röntgenbestrahlung mit $800 \mathrm{r}, 0-30$ Min. nach i.p.-Injektion von $4 \mu$ C $2 \cdot{ }^{14} \mathrm{C}$-Glycin $\left(=5,32 \cdot 10^{6} \mathrm{cpm}\right)$. - - bestrahlt mit $800 \mathrm{r}$, ० - - ० Kontrollen.

erklären ist, in denen möglicherweise die Proteinsynthese inhibiert wird. Vielmehr sprechen diese Befunde dafür, daß es in den bestrahlten Tieren zu einer rascheren Resorption der injizierten markierten Aminosäure kommt. Hinweise hierfür ergeben sich aus Untersuchungs mit ${ }^{32} \mathrm{P}$-Ortho-Phosphat. Sherman und Forssberg ${ }^{17}$ konnten unmittelbar nach Ganzkörperbestrahlung mit $800 \mathrm{R}$ einen um $40 \%$ erhöhten Einbau von ${ }^{32} \mathrm{P}$-Ortho-Phosphat in die Mäuseleber nachweisen. Dieser Befund deutet auf eine starke Zunahme der Permeabilität gegenüber Phosphat-Ionen hin. Der Effekt wird weniger deutlich bei längerem Zeitintervall. Die PermeabilitätsSteigerung betrifft aber offensichtlich insbesondere das Peritoneum. Wurde nämlich die Leber in vitro bestrahlt, so fand sich keine Zunahme der ${ }^{32} \mathrm{P}$-Aktivität in den Leberstückchen. Eine gesteigerte Kapillarpermeabilität 14 bis 72 Stdn. nach Abdomenbestrahlung von Ratten mit $1500 \mathrm{R}$ ließ sich durch Austritt von Protein-gebundenem Trypanblau im Dünndarm nachweisen (WILloughby ${ }^{18}$ ). Auch zahlreiche Untersuchungen über Elektrolytverschiebungen in bestrahlten Tieren und an isolierten Tumorzellen weisen darauf hin, daß es in bestrahlten Zellen zu Permeations-Steigerungen kommt (s. BAcQ und Alexander ${ }^{19}$ ).

16 H. Goetze u. E. Goetze, Acta biol. med. german. 15, 353 [1965].

17 F. G. Sherman u. A. Forssberg, Arch. Biochemistry 48, 293 [1954].

18 D. A. Willoughby, J. Physiol. 148, 42 [1959].

19 Z. M. Bace u. P. Alexander, Fundamentals of Radiobiology Pergamon Press, London 1961. 